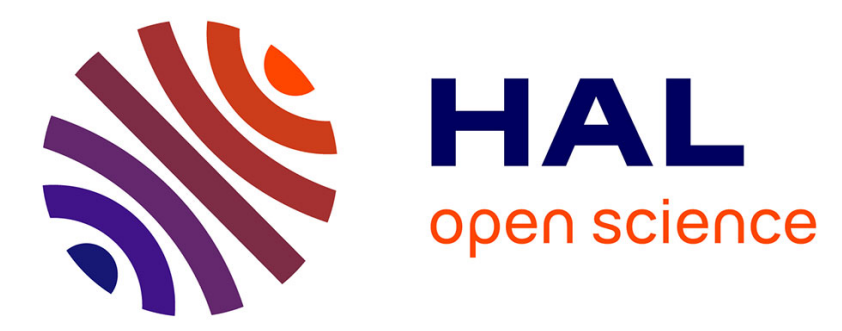

\title{
Éléments de topographie chrétienne à Genève (Suisse)
}

Charles Bonnet

\section{To cite this version:}

Charles Bonnet. Éléments de topographie chrétienne à Genève (Suisse). Gallia - Archéologie de la France antique, 2006, Antiquité tardive, haut Moyen Âge et premiers temps chrétiens en Gaule méridionale (première partie) : réseau des cités, monde urbain et monde des morts, 63, pp.111-115. 10.3406/galia.2006.3288 . hal-01914307

\section{HAL Id: hal-01914307 \\ https://hal.science/hal-01914307}

Submitted on 8 Jan 2020

HAL is a multi-disciplinary open access archive for the deposit and dissemination of scientific research documents, whether they are published or not. The documents may come from teaching and research institutions in France or abroad, or from public or private research centers.
L'archive ouverte pluridisciplinaire HAL, est destinée au dépôt et à la diffusion de documents scientifiques de niveau recherche, publiés ou non, émanant des établissements d'enseignement et de recherche français ou étrangers, des laboratoires publics ou privés.

\section{(ㅇ)(1) $\$$}

Distributed under a Creative Commons Attribution - NonCommercial - NoDerivatives 44.0 


\title{
ÉLÉMENTS DE TOPOGRAPHIE CHRÉTIENNE À GENÈVE (SUISSE)
}

\author{
Charles BONNET
}

\begin{abstract}
Mots-clés. Groupe épiscopal, cathédrales, atrium, rois burgondes, églises suburbaines.
Résumé. Les fouilles archéologiques menées durant 40 ans dans la ville de Genève ont permis de mettre au jour les vestiges d'un espace urbain que l'on suit de la fin du II ${ }^{e}$ s. av. J.-C. jusqu'aux époques récentes. Le chantier de la cathédrale Saint-Pierre a révélé l'évolution architecturale du groupe épiscopal avec ses églises aux fonctions diversifiées et de nombreux bâtiments annexes. Les recherches menées dans plusieurs lieux de culte hors les murs ont également apporté une image remarquable de la topographie chrétienne.
\end{abstract}

Key-words. Bishopric, cathedrals, atrium, Burgundian kings, suburban churches.

Abstract. During the archaeological excavations carried out for 40 years in the town of Geneva, the remains of an urban area have been detected from the end of the $2^{\text {nd }} c$. BC to modern times. In the site of Saint-Peter cathedral, the architectural development of the bishopric has been observed, having varied purposes and numerous adjacent buildings. Investigations inside several cult places outside the walls have also brought an outstanding insight of the Christian topography.

Translation: Isabelle FAUDUET

Schlagwörter. Kirchenfamilie, Kathedralen, atrium, Burgunderkönige, Kirchen außerhalb der Stadt.

Zusammenfassung. Eine vierzigjährige Ausgrabungstätigkeit ermöglichte in Genf die Freilegung eines städtischen Siedlungsbereichs, der sich vom Ende des zweiten Jahrhunderts v. Chr. bis in die Neuzeit verfolgen läßt. Durch die Ausgrabungen an der Kathedrale SaintPierre wurde die bauliche Entwicklung der ersten Kirchenfamilie mit ihren Kirchenbauten unterschiedlicher Funktion und zahlreichen Nebengebäuden erschlossen. Die Untersuchungen in verschiedenen Anlagen extra muros liefern ebenfalls einen außergewöhnlichen Aspekt zur christlichen Topographie.

Übersetzung: Stefan WIRTH

Les recherches systématiques du Service cantonal d'archéologie de l'État ont apporté une riche documentation sur les bâtiments paléochrétiens de Genève, ravivant l'intérêt des habitants pour l'histoire de leur ville (fig. 54).

La restauration de la cathédrale Saint-Pierre a été l'occasion de soumettre aux autorités un projet de recherches à très long terme. C'est ainsi que durant trente années, une équipe scientifique a effectué, sans interruption, un patient travail d'étude et de mise en valeur d'un site qui, à ce jour, reste exceptionnel. La présentation rend compte des premiers états de l'occupation humaine sur la colline formée entre le lit du Rhône et celui de l'Arve, rivière qui descend du Mont-Blanc. Il existait, vers 123 av. J.-C., une place publique, un atelier de potier et des constructions en terre et en bois se rattachant à une habitation aristocratique. Sous la partie orientale de la terrasse morainique s'étendait encore une aire funéraire; une des tombes continuera d'être vénérée dans les constructions successives établies ensuite à cet emplacement. Il semble que l'on en conserve encore indirectement le souvenir lors de l'édification d'une église primitive dont la large abside outrepassée pourrait attester un culte des reliques.

La mention, vers l'an 400, d'un évêque Isaac, alors déjà âgé, confirme l'existence d'une christianisation précoce. Une vingtaine d'années auparavant, l'ecclésiastique disposait déjà d'un vaste complexe architectural, développé 


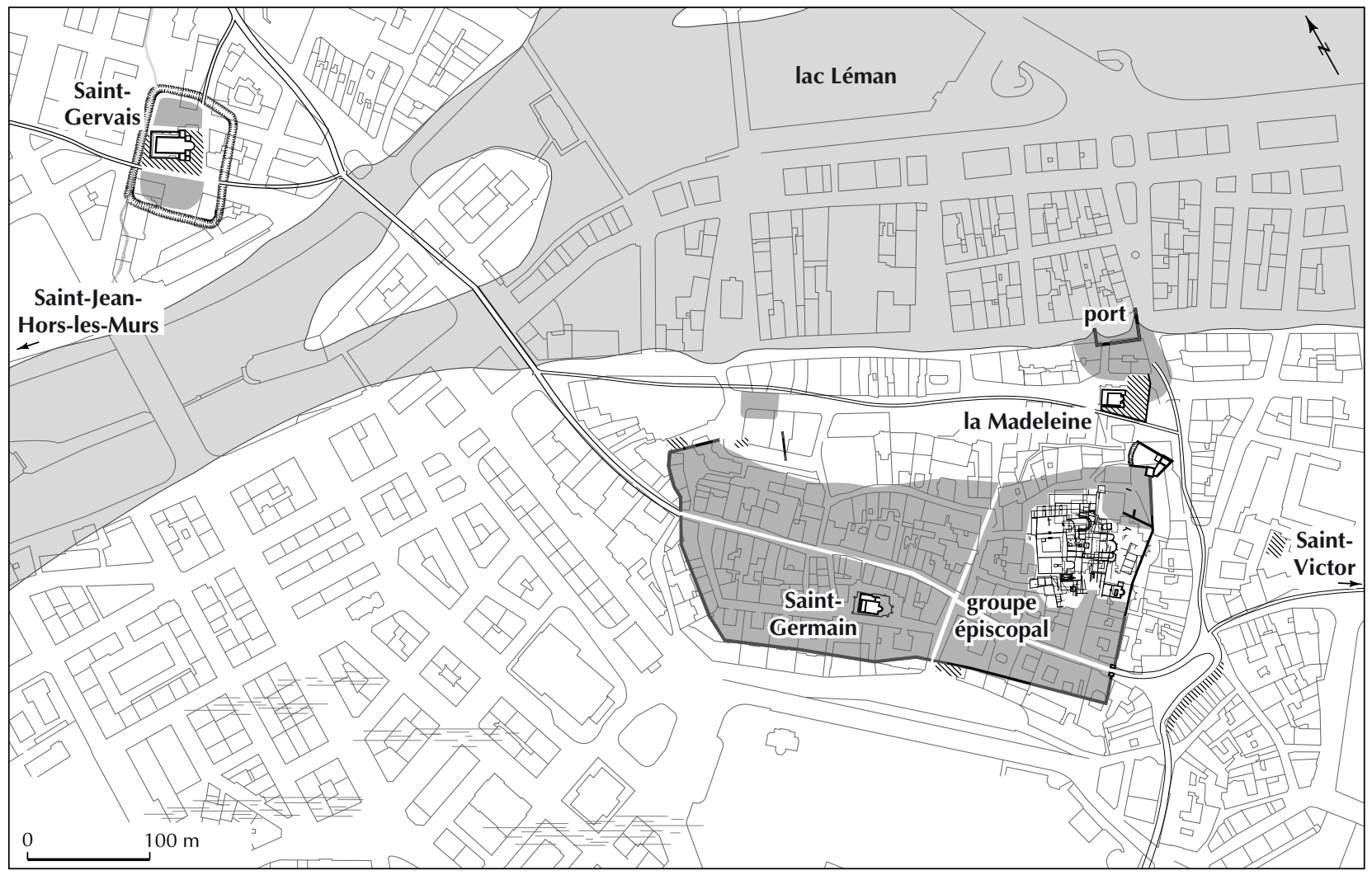

Fig. 54 - Genève (Suisse). Plan topographique de la ville durant le haut Moyen Âge (DAO A. Peillex et M. Berti, Service cantonal d'archéologie, Genève).

progressivement au détriment d'un grand bâtiment résidentiel appartenant sans doute à l'un des responsables de la ville (fig. $55, \mathrm{n}^{\mathrm{O}} 1$ ). La première cathédrale, de près de $25 \mathrm{~m}$ de longueur, est construite en opus africanum; des cellules chauffées, réservées à des reclus, s'adossent à sa paroi latérale nord. De l'autre côté, une galerie donne accès au baptistère dont la cuve carrée est placée au nord, le long de l'axe menant à l'abside outrepassée. Dans les deux monuments, des barrières séparent l'espace liturgique de celui des fidèles; contre certains murs, l'emplacement de bancs en bois a été localisé. Au sud-est, selon une disposition en biais, se trouve la curieuse église formée d'une nef étroite et d'une grande abside outrepassée; son plan est presque identique à celui de la première église de Martigny. C'est au pied de l'enceinte du Bas-Empire que sont établies la résidence de l'évêque ainsi que son église privée, qui paraît prolonger l'alignement des édifices religieux.

Cette implantation de la seconde moitié $d u I^{\mathrm{e}} \mathrm{s}$. a bien évidemment modifié le parcellaire précédent, datant de la paix romaine; des terrasses ont notamment été créées à l'intérieur du système fortifié. Seul le bâtiment rési- dentiel, qui était peut-être le praetorium, est partiellement maintenu puisque deux de ses ailes sont encore habitées. À côté, on note la présence d'une construction rectangulaire, singularisée par une porte monumentale percée au travers de sa façade occidentale. Dans ses premiers niveaux d'occupation, des centaines de monnaies semblent correspondre à des sortes d'ex voto; on aurait à cet endroit un emplacement consacré qui reste à interpréter. Plusieurs salles de réception, certaines chauffées, sont disposées au centre du groupe épiscopal ainsi constitué. Comme celles de la résidence de l'évêque, elles devaient servir à l'accueil des visiteurs et aux réunions de clercs. Un triclinium et sa boucherie attestent aussi de repas pris en commun. Les multiples fonctions liées à la charge de l'évêque rendaient toutes ces salles indispensables. Comme on peut le constater, les maîtres d'œuvre ont su conserver une certaine unité à ce quartier religieux composé de multiples constructions.

Au cours du V $\mathrm{V}^{\mathrm{e}}$ s., l'ensemble est modifié par l'adjonction d'un atrium et d'une deuxième cathédrale au sud, de proportions presque semblables à celles de la cathédrale nord (fig. $55, \mathrm{n}^{\circ} 2$ ). On relèvera que les installations liturgiques 


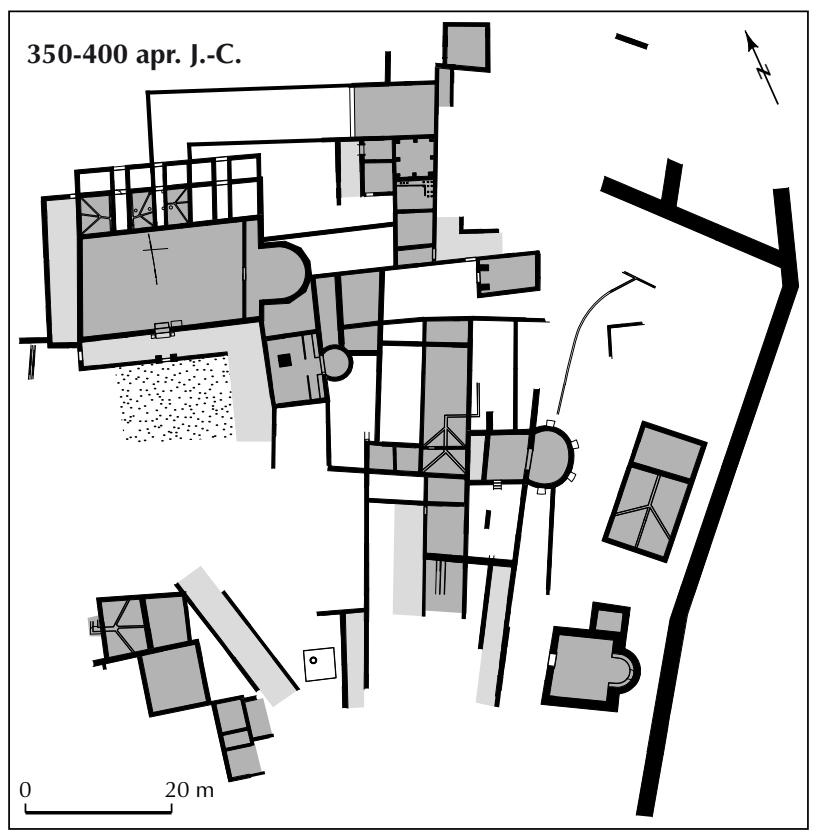

1

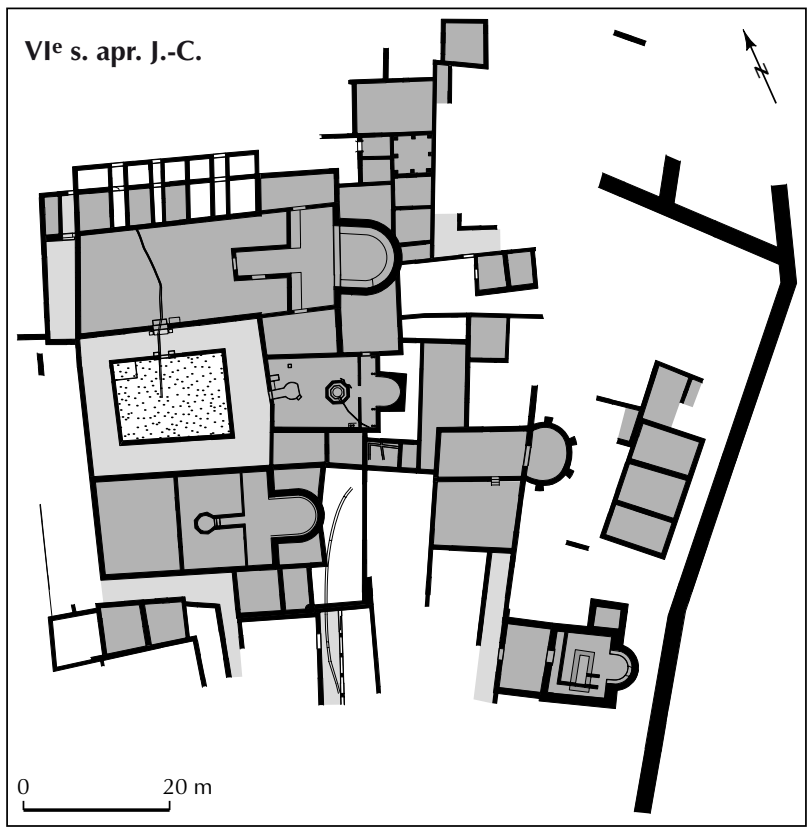

3
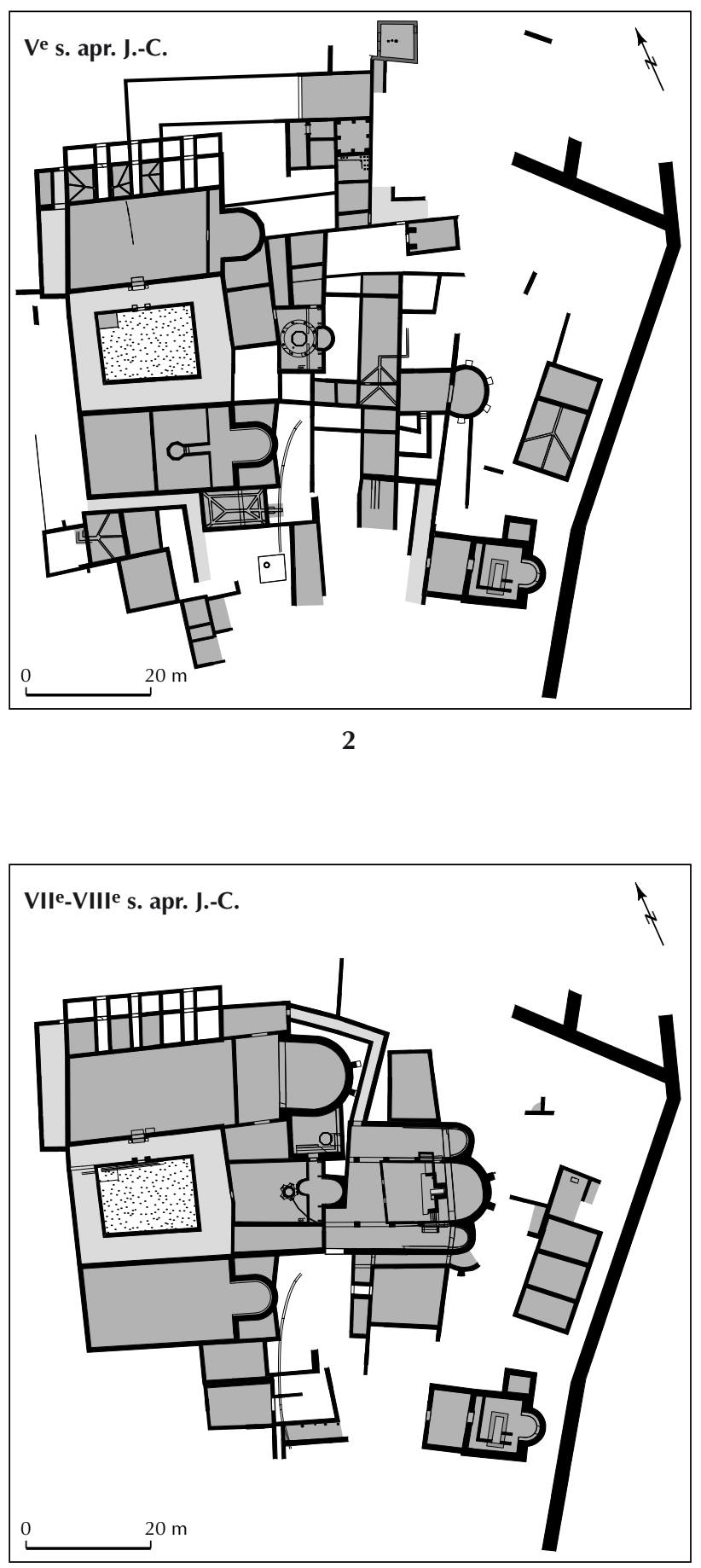

4

$\square$ mur $\square$ sol $\square$ galerie $\square$ cour

Fig. 55 - Genève (Suisse). Le groupe épiscopal du IV s. au VIII $s$.

(DAO A. Peillex et M. Berti, Service cantonal d'archéologie, Genève). 
permettent au desservant de lire les textes anciens depuis un ambon polygonal et sans doute d'expliquer la nouvelle religion. Une salle de réception remarquable est ajoutée contre le chœur du nouvel édifice; elle se distingue par ses dimensions généreuses, son décor - peintures murales et mosaïque de sol -, et son système de chauffage en soussol. Le baptistère est recentré entre les deux sanctuaires et pourvu d'un ciborium monumental. Des transformations sont également apportées dans le bâtiment résidentiel romain, qui a donc conservé un certain prestige et fait toujours partie du quartier religieux. Le plan général du groupe épiscopal tel qu'il a été restitué atteste une organisation clairement définie, issue d'un pouvoir bien implanté dans la ville. S'il s'inscrit dans une tradition ancienne, il ne cessera d'être l'objet de modifications : les bâtiments qui le constituent sont régulièrement restaurés, agrandis ou embellis. L'abondance de fragments de peintures murales, de stucs et de mosaïques donne une idée de la richesse des décors mis en œuvre.

Vers l'an 500, des guerres fratricides entre rois burgondes provoquent un incendie et de violentes destructions dans cette partie de la civitas. Les travaux menés après ces événements vont être relativement ambitieux (fig. 55, no 3). Le chœur de la cathédrale nord est notablement agrandi et flanqué de larges annexes lui conférant un caractère cruciforme. La solea qui prolonge l'espace liturgique aboutit à une porte, sans doute utilisée lors de cérémonies processionnelles. Une fois encore, le baptistère est rebâti, mais cette fois selon un plan plus allongé vers l'ouest; la cuve, déplacée, est surmontée d'un ciborium octogonal. L'église à large abside outrepassée voit sa nef allongée, tandis qu'une grande salle de réception la jouxte du côté sud. À côté, l'église épiscopale fait également l'objet de remaniements. Le type des maçonneries qui jusqu'ici relevait de techniques antiques subit de profonds changements : les murs sont plus épais et les sols de terrazzo sont remplacés par d'épaisses couches de terre argileuse.

Durant la fin du haut Moyen Âge, le groupe épiscopal reçoit une parure monumentale qui répond mieux à l'évolution de la liturgie (fig. 55, no 4 ). Les différents édifices sont souvent restaurés et une seconde résidence épiscopale est bâtie au sud. Toutefois, on observe un abandon progressif des principaux sanctuaires au profit d'une cathédrale unique et d'un ensemble canonial. L'église à large abside, vraisemblablement dédiée au culte du souvenir de personnages vénérés, paraît donner naissance à une cathédrale à trois nefs dans laquelle est ménagée une circulation facilitant l'accès vers une tombe exceptionnelle et vers des emplacements privilégiés secondaires dans les bas-côtés. Après un certain temps, l'église va s'étendre sur toute la surface du baptistère, qui a perdu ses fonctions. Peu après l'an mil, une crypte circulaire est édifiée hors œuvre, derrière le chevet; elle sera englobée dans un nouveau chœur roman à la fin du $\mathrm{XI}^{\mathrm{e}} \mathrm{s}$. Cette extraordinaire continuité ne sera pas interrompue pendant le Moyen Âge, ni même après la Réforme de 1536.

À la topographie chrétienne étudiée intra muros, il convient encore d'inclure la petite église de Saint-Germain, pourvue d'annexes qui n'ont été que partiellement reconnues (fig. 54). Cet édifice a été considéré comme le siège de l'une des paroisses primitives à l'intérieur de la ville fortifiée. Les fragments de son chancel de la fin $\mathrm{du} \mathrm{IV}^{\mathrm{e}}$ s. ou du V $\mathrm{V}^{\mathrm{e}}$ s. sont d'un grand intérêt avec la représentation en relief de cerfs et d'agneaux s'affrontant de part et d'autre d'un motif central surmonté d'une croix latine. Ces éléments sculptés dans un calcaire fin provenant d'une carrière proche de Genève sont vraisemblablement issus d'un atelier local.

Hors les murs, plusieurs aires funéraires sont aujourd'hui mieux documentées. Dans le quartier du port, le sanctuaire de la Madeleine s'élevait à l'emplacement d'une nécropole du Bas-Empire comportant plusieurs sépultures privilégiées associées à de petits bâtiments funéraires. C'est d'ailleurs une memoria, dotée d'un autel, qui est à l'origine de l'église dans laquelle les inhumations se sont multipliées. Les tombes maçonnées, ou à dalles, et les sarcophages occupant bientôt toute la surface disponible, la nef est élargie par une galerie menant vers une annexe proche de l'abside pour permettre de nouvelles inhumations. Le monument connaîtra encore bien d'autres transformations au cours du Moyen Âge; il devient le siège d'une paroisse de la ville basse $\mathrm{au} \mathrm{XI}{ }^{\mathrm{e}} \mathrm{s}$.

Une fondation de la reine burgonde Sédeleude paraît attestée à la fin $\mathrm{du} \mathrm{V}^{\mathrm{e}} \mathrm{s}$. sur la rive gauche du lac et du Rhône. Elle a donné naissance au célèbre prieuré de Saint-Victor dépendant de Cluny. Il ne reste pratiquement rien de ce vaste complexe, ni de la chapelle Saint-Laurent détruite par des fortifications tardives. Sur la rive droite, l'église de Saint-Gervais occupe l'emplacement d'un site du Néolithique moyen ancien, sur lequel sont ensuite installés un alignement de menhirs et des tombes du Bronze final. Puis, un lieu de culte romain se développe; un grand mausolée s'implante au $\mathrm{IV}^{\mathrm{e}} \mathrm{s}$., dont quelques blocs ont été retrouvés en remploi dans la voûte de la crypte d'une église cruciforme bâtie plus tard sur l'emplacement consacré. L'église paléochrétienne de Saint-Gervais, mise en évidence lors de fouilles systématiques, est de dimensions tout à 
fait exceptionnelles. La nef centrale était entourée sur trois côtés par des galeries prévues pour des inhumations. $\mathrm{Au}$ nord-est, une annexe, dotée d'une abside au $\mathrm{VI}^{\mathrm{e}} \mathrm{s}$, se distingue par des tombeaux monumentaux d'un type très rare dans la région. Notons encore les aménagements liturgiques destinés à protéger les escaliers conduisant à la crypte ou au chœur surélevé.

En aval, au bord du Rhône, des grottes, peut-être celles mentionnées dans la relation d'un miracle de saint Romain à Genève, ont favorisé la construction d'une église en bois très ancienne. Plusieurs sanctuaires du haut Moyen Âge témoignent en zone rurale d'une longue tradition de cette architecture en bois. Ce n'est que peu avant l'an mil que l'on transforme cette église de Saint-Jean-Hors-les-Murs en un imposant bâtiment en pierre, où des tombes maçonnées sont aménagées pour les ecclésiastiques d'une abbaye. La topographie de la cité s'articule ainsi autour de différents monuments religieux dont l'architecture révèle plusieurs courants d'influence. Les analyses détaillées qui ont pu être menées par le Service cantonal d'archéologie de l'État de Genève ont livré une information renouvelée sur des sites où la continuité est presque toujours bien attestée, contribuant ainsi à une meilleure perception d'une période relativement mal connue dans le massif alpin. 